\title{
BMJ Open Antithrombotic therapy in patients receiving saphenous vein coronary artery bypass grafts: a protocol for a systematic review and network meta- analysis
}

\author{
Karla Solo, ${ }^{1}$ Janet Martin, ${ }^{1,2}$ Shahar Lavi, ${ }^{3}$ Conrad Kabali, ${ }^{4}$ Ava John-Baptiste,,${ }^{1,2,5}$ \\ Immaculate F Nevis, ${ }^{6}$ Tawfiq Choudhury, ${ }^{3}$ Mamas A Mamas, ${ }^{7}$ Rodrigo Bagur ${ }^{1,3,7}$
}

To cite: Solo K, Martin J, Lavi S, et al. Antithrombotic therapy in patients receiving saphenous vein coronary artery bypass grafts: a protocol for a systematic review and network meta-analysis. BMJ Open 2018;8:e019555. doi:10.1136/ bmjopen-2017-019555

- Prepublication history and additional material for this paper are available online. To view these files, please visit the journal online (http://dx.doi. org/10.1136/bmjopen-2017019555).

Received 11 September 2017 Revised 15 February 2018 Accepted 9 March 2018

Check for updates

For numbered affiliations see end of article.

Correspondence to

Dr Rodrigo Bagur;

rodrigobagur@yahoo.com

\section{ABSTRACT}

Introduction The current evidence for the prevention of saphenous vein graft failure (SVGF) after coronary artery bypass graft (CABG) surgery consists of direct head-tohead comparison of treatments (including placebo) in randomised-controlled trials (RCTs) and observational studies. However, summarising the evidence using traditional pairwise meta-analyses does not allow the inclusion of data from treatments that have not been compared head to head. Exclusion of such comparisons could impact the precision of pooled estimates in a metaanalysis. Hence, to address the challenge of whether aspirin alone or in addition to another antithrombotic agent is a more effective regimen to improve SVG patency, a network meta-analysis (NMA) is necessary. The objectives of this study are to synthesise the available evidence on antithrombotic agents (or their combination) and estimate the treatment effects among direct and indirect treatment comparisons on SVGF and major adverse cardiovascular events, and to generate a treatment ranking according to their efficacy and safety outcomes.

Methods We will perform a systematic review of RCTs evaluating antithrombotic agents in patients undergoing CABG. A comprehensive English literature search will be conducted using electronic databases and grey literature resources to identify published and unpublished articles. Two individuals will independently and in duplicate screen potential studies, assess the eligibility of potential studies and extract data. Risk of bias and quality of evidence will also be evaluated independently and in duplicate. We will investigate the data to ensure its suitability for NMA, including adequacy of the outcome data and transitivity of treatment effects. We plan to estimate the pooled direct, indirect and the mixed effects for all antithrombotic agents using a NMA.

Ethics and dissemination Due to the nature of the study, there are no ethical concerns nor informed consent required. We anticipate that this NMA will be the first to simultaneously assess the relative effects of multiple antithrombotic agents in patients undergoing CABG. The results of this NMA will inform clinicians, patients and guideline developers the best available evidence on comparative effects benefits of antithrombotic agents after CABG while considering the side effect profile to support

\section{Strengths and limitations of this study}

- This review is anticipated to be the first network meta-analysis to evaluate the comparative effects of multiple antithrombotic agents in patients undergoing coronary artery bypass graft.

- The strengths of this review are the comprehensive literature search strategy, restriction of studies to randomised controlled trials, duplicate assessment of eligibility, risk of bias and data abstraction. We will also use the Grade of Recommendations Assessment, Development and Evaluation system to rate the quality of evidence.

- This study will be limited to trials that assessed the effects of antithrombotic agents on saphenous vein graft failure. A high rate of loss to follow-up is therefore expected.

future clinical decision-making. We will disseminate the results of our systematic review and NMA through a peerreviewed journal.

PROSPERO registration number CRD42017065678.

\section{INTRODUCTION}

Despite the remarkable advance in percutaneous coronary intervention (PCI) and drug-eluting stents, coronary artery bypass grafting $(\mathrm{CABG})$ is still the preferred treatment for patients presenting with multivessel coronary artery disease. ${ }^{2}$ However, CABG patients remain at risk for subsequent major adverse cardiovascular events (MACE) and saphenous vein graft failure (SVGF) ${ }^{3-5}$ SVGs are the most commonly used conduits and previous studies reported that $15 \%$ to $30 \%$ of SVGs occlude within the first year after $\mathrm{CABG}^{6}$ and approximately $40 \%$ to $60 \%$ at 10 years after surgery. ${ }^{7-9}$

The use of acetylsalicylic acid (aspirin) has been associated with improved 
clinical outcomes and reduction in SVGF after CABG surgery. ${ }^{1}{ }^{10-17}$ Antiplatelet agents other than aspirin (ie, clopidogrel) have been reported as an alternative option, although with conflicting efficacy in the literature. ${ }^{18-22} \mathrm{In}$ addition, medications that inhibit venous thrombi formation, such as oral anticoagulants (OAC), have also been tested in clinical trials to provide protection against SVGF but with an increased risk of bleeding as a trade-off. ${ }^{1723}$

Thus far, the evidence for the prevention of SVGF consists of direct head-to-head comparison of treatments (including placebo) in randomised-controlled trials (RCTs) and observational studies. This poses a practical challenge to clinicians for choosing therapeutic alternatives because a direct comparison is rarely seen or not available for many antithrombotic agents. In addition, summarising evidence using the traditional pairwise meta-analyses would not allow for the inclusion of data from treatments that have not been compared head-tohead and thus, the results from indirect combined with direct evidence can improve precision for treatments that have been directly evaluated. ${ }^{24}$ Therefore, to address the challenge of whether aspirin alone, or in addition to another antithrombotic agent is a more effective regimen to improve SVG patency, a network meta-analysis (NMA) is necessary. Hence, the objectives of this study are to synthesise the available evidence on antithrombotic agents (or their combination) and estimate the treatment effects among direct and indirect treatment comparisons on SVGF and MACE, and to generate a treatment ranking according to their efficacy and safety outcomes using a NMA.

\section{METHODS AND DESIGN}

This systematic review will be conducted according to the Cochrane Collaboration recommendations, ${ }^{25}$ and will adhere to the Preferred Reporting Items for Systematic Reviews and Meta-Analyses (PRISMA) Extension for NMAs of healthcare interventions guidelines (online supplementary material) ${ }^{26}$ Our systematic review and NMA protocol has been registered in the International Prospective Register for Systematic Reviews (PROSPERO: CRD42017065678).

\section{Eligibility criteria}

The eligibility criteria are pre-specified according to the $\operatorname{PICO}(\mathrm{S})$ criteria.

\section{Types of participants}

Participants will include adults $\geq 18$ years undergoing CABG surgery and receiving at least one SVG. To increase the likelihood of transitivity assumption being met, we will exclude studies that included participants with clinical conditions that prevent them from receiving one or more of the above treatments. For example, we will exclude studies including participants with thromboembolic disease or undergoing concomitant mechanical valve replacement needing life-long OAC, or those comparing clopidogrel to placebo in patients with a contraindication for aspirin (ie, allergy) or warfarin (ie, prior major bleeding).

\section{Types of interventions}

The interventions are aspirin, clopidogrel, prasugrel, ticagrelor, vitamin $\mathrm{K}$ antagonists (warfarin, acenocoumarol or phenprocoumon), new OAC (rivaroxaban, apixaban, dabigatran) and placebo, regardless of drug regimen. We will assess the comparability of any other antithrombotic agents that we are not aware of, with those stated above, and then will consider them eligible if they are still used in clinical practice. The comparator can be placebo, inactive control or a different oral antithrombotic agent. Comparing interventions of the same drug with different regimens is beyond the scope of this study.

\section{Types of outcome}

Included studies must report SVGF as one of the endpoints (defined below).

- Primary outcome: SVGF as per-patient analysis;

- Secondary efficacy outcomes: all-cause mortality, any stroke (ischaemic or haemorrhagic) or transient ischaemic attack, any myocardial infarction (fatal or non-fatal) and major bleeding;

- Secondary safety outcomes: intracranial haemorrhage, life-threatening bleeding, minor bleeding, red blood cells transfusion, MACE and hospital readmission due to cardiovascular cause.

We anticipate study authors will use a variety of definitions for bleeding and for SVGF. If several definitions of major bleeding are presented in the same study, our preferred definition will be according to the Thrombolysis In Myocardial Infarction criteria. ${ }^{27}$ Our preferred definition for SVGF will be total occlusion (100\%) as detected by invasive angiography or non-invasive CT assessment on a per-patient basis (ie, patients with at least one occluded SVG). If the study does not provide data corresponding to the preferred definition of SVGF, we will incorporate study data reported in the following way, based on this priority sequence:

- Any SVG stenosis deemed to require treatment with PCI on a per-patient basis;

- Cardiac reintervention (defined as the need for SVG-related PCI or repeat CABG);

- SVG stenosis $>70 \%$ on a per-patient basis;

- SVG stenosis $>70 \%$ on a per-graft basis;

- Any SVG stenosis deemed to be treated with PCI on a per-graft basis.

In addition, when available, data on perioperative SVGF will be excluded from the analysis as such SVGF occurs mainly due to technical factors (eg, mechanical trauma, poor distal run-off), regardless of the intervention received, resulting in underestimation of the results. The remaining outcomes will be defined per study author definition. Finally, and based on available data, we will categorise outcomes on reported timelines of follow-up as short, medium and long term. 
Types of studies

Eligible studies are RCTs assessing the effect of oral antithrombotic agents on SVGF after CABG.

\section{Search strategy}

A comprehensive search in English literature will be conducted using the following electronic databases: Medline, Embase, Web of Science, Cumulative Index of Nursing and Allied Health and Cochrane Library to identify published articles on antithrombotic therapy following CABG, from inception to November 2017. In consultation with a medical librarian, the search strategy will be created to combine relevant Medical Subject Headings $(\mathrm{MeSH})$ and keywords with synonyms and names of generic and brand names of the antithrombotic agents. The search will not be restricted by year of publication. Additionally, we will perform a grey literature search using trial registries (eg, ClinicalTrials.gov), regulatory agency databases, drug companies, dissertations and conference proceedings for unpublished studies. We will also check reference lists of included RCTs and relevant systematic reviews. Monthly search alerts will be set up for electronic databases to identify any new published articles until the submission of the present study. Contact with experts in the field of interest will also be sought to inquire about unpublished studies. A detailed search strategy for all databases including search concepts, MeSH term, keywords and Boolean operators is presented within the online supplementary material. Non-English language studies and ongoing RCTs without extractable data will be excluded. Duplicate publications of original research will be also excluded. If the same population was used in multiple studies, the article with the longest follow-up will be included.

\section{Study selection}

To assess study eligibility, all title/abstracts and full-text articles will be independently screened by two reviewers and disagreements will be resolved by a third reviewer. If necessary, methodological experts will be consulted to reach consensus. Eligible articles will be selected according to prespecified inclusion criteria and the selection process is shown in online supplementary figure 1.

\section{Data extraction}

The study data will be extracted independently and in duplicate from eligible studies and will include study and publication details, participants and intervention characteristics as well as outcome data (table 1). Whenever possible, we will extract arm-level data (ie, number of events) from the included studies, otherwise, contrastlevel data (ie, effect sizes and standard errors of a direct comparison) will be used. The study data will be collected using a predetermined data collection template, which will be pilot tested on a few randomly- elected included studies. In case of insufficient information, the study authors will be contacted to provide further information. Two independent reviewers will perform data extraction and in case of discrepancies, a third reviewer will be consulted. We will extract arm-level data whenever possible.

\section{Risk of bias assessment}

Risk of bias in the eligible trials will be assessed using the updated Cochrane Collaboration Risk of Bias Tool (RoB V.2.0), ${ }^{28}$ which consists of five domains of bias that are relevant to the quality of RCTs. The five domains include bias due to the randomisation process, deviation from intended intervention, incomplete outcome data, measurement of the outcome, and selective reporting.

Table 1 Data extraction template

$\begin{array}{ll}\text { Publication details } & \begin{array}{l}\text { First author, country of conduct, funding sources (for profit, mixed and non-profit) and year of } \\ \text { publication. }\end{array} \\ \begin{array}{l}\text { Study } \\ \text { characteristics }\end{array} & \begin{array}{l}\text { Study design (randomised or non-randomised), setting (single or multicentre), accrual period, } \\ \text { length of follow-up, inclusion and exclusion criteria, Cochrane risk of bias, rates of loss to follow- } \\ \text { up (with reasons), number of study arms, number of patients randomised per arm, number of } \\ \text { patients analysed per arm, antifibrinolytic use during surgery (eg, aprotinin or tranexamic acid) } \\ \text { and surgical technique (eg, endarterectomy or not, off pump or on pump, endoscopic vein } \\ \text { harvesting or open harvesting, single or multiple vein grafts, sequential grafts, 'no-touch' grafting } \\ \text { or conventional) }\end{array} \\ \text { Participant characteristics } & \begin{array}{l}\text { Age; proportion of women; proportion of patients with atrial fibrillation or flutter, hyperlipidaemia, } \\ \text { prior MI, prior TIA/stroke, heart failure (NYHA functional class III-IV), diabetes mellitus, } \\ \text { hypertension or chronic kidney disease; number of vessel grafted, left ventricular ejection fraction }\end{array} \\ \text { Intervention characteristics } & \begin{array}{l}\text { Dose, frequency, duration, timing for the start of treatment and adherence } \\ \text { Outcomes of Interest }\end{array} \\ \begin{array}{l}\text { Number of patients with SVGF, major bleeding, any stroke or TIA, any MI, heart failure, intracranial } \\ \text { haemorrhage; number patients needed RBCs transfusions; number of patients admitted to } \\ \text { hospital due to cardiovascular cause; number of deaths; number of occluded SVGs; authors' } \\ \text { definitions of outcomes; time and method for outcome assessments and any subgroup analyses }\end{array}\end{array}$

MI, myocardial infarction; NYHA, New York Heart Association; RBCs, red blood cells; SVGF, saphenous vein graft failure; TIA, transient ischaemic attack. 
Each domain will be assigned a score of 'high risk' (if the study is at high risk of bias for more than one domain or some concerns for multiple domains), 'low risk' (if the study is at low risk of bias for all domains) or 'some concern' (if the study has some concern for at least one domain) depending on the degree of bias on an outcome level.

\section{Rating the confidence in estimates of the effect in the network meta-analysis}

Once the NMA is completed, we will then appraise the quality of treatment effect estimates using the Grade of Recommendations Assessment, Development and Evaluation (GRADE) working group for NMA. ${ }^{29}$ We will follow the four-step approach ${ }^{29}$ : once we obtain direct and indirect treatment estimates, we will rate the quality of evidence from the direct and indirect comparisons using the five GRADE categories: risk of bias, inconsistency, indirectness, imprecision and publication bias. We will then rate the quality of evidence of the NMA effect estimate.

\section{Measures of treatment effect}

The primary unit of analysis will be based on per-patient outcomes measures. If per-patient data were not available in all included studies, the unit of analysis will depend on the consistency of the results (magnitude and direction of effect estimates) between the per-patient versus per-graft meta-analysis for all direct treatment comparisons. If these meta analyses yield different effects, we will conduct a separate analysis for each level; however, if there is no important difference in effect sizes (ie, large overlaps in the $95 \%$ CI of effect sizes) between units of analysis for all comparisons, we will pool data from studies that reported the per-patient data with those that reported only the per-graft data (accounting for clustering effects). We will then report results generating an inference at patientlevel analysis as it is clinically relevant to apply the intervention to the patient.

\section{Methods for indirect and mixed comparisons}

We will present indirect comparisons of antithrombotic agents using a network diagram for outcomes that have at least 10 studies. If there are any trials that formed a node, which are not connected to the rest of the network, we will exclude them. The common comparator will be aspirin as it is the current standard of care. ${ }^{1217}$ To assess whether the transitivity assumption (ie, the distribution of treatment response is similar across all RCTs) holds, we will qualitatively assess the distribution of patient and study characteristics that modify treatment effects (ie, effect modifiers; see subgroup analysis and metaregression) across treatment comparisons by presenting tabulated results of these characteristics. We will also evaluate the statistical manifestation of the assumption, known as consistency assumption by performing a global test for inconsistency (design-by-treatment interaction model) ${ }^{30}$ and a local test (inconsistency plot) ${ }^{31}$ to identify sources of inconsistency within every closed loop in the network. In case the transitivity assumption is violated, methods for covariate adjustment will be considered. ${ }^{32} 33$

The NMA will be based on a mixed treatment comparison model generation using the network commands package (Stata V.14.0, StataCorp) ${ }^{34}$ We will conduct a frequentist hierarchical random-effects model for conducting our NMA. The OR will be reported along with $95 \%$ CIs. A network diagram will be generated to show the size of the different trials and the weight that it contributes towards the point estimate. $\mathrm{P}<0.05$ will be considered statistically significant.

\section{Treatment ranking}

To estimate the percentage of efficacy of each antithrombotic agent for being at each possible rank, we will use the surface under the cumulative ranking curve (SUCRA). ${ }^{31} 35$ The higher the SUCRA score, the more efficacious the treatment is expected to be.

\section{Studies with multiple treatment groups}

Multiarm studies will be included and will be treated as multiple independent two-arm studies in pairwise meta-analyses. In the NMA, we will include such studies and account for the correlations between effect sizes induced by multiarm studies using a multivariate approach. ${ }^{36}$

\section{Dealing with missing data}

We will attempt to contact study authors to obtain missing data. We will also discuss reasons for missing data as well as its extent and nature and how missing data was handled by each study. We will conduct sensitivity analyses, such as best-worst case scenario analysis for dichotomous outcome, to explore the potential impact of the missing data. Since we anticipate that some patients will be lost to follow-up, and some studies will ascertain angiographic follow-up on only a subset of the whole population, we will also record the 'loss to follow-up' to inform sensitivity analyses using different assumptions about the missing data (see sensitivity analysis). We will extract data from studies that report outcomes using the intention-to-treat principle, that is, we will analyse all participants according to the treatment they were randomised to, as long as the deviation from the protocol is negligible.

\section{Assessment of reporting bias}

When a sufficient number of studies is available (at least 10 trials) for each direct treatment comparison and outcome, we will assess the possibility of reporting bias by visually examining the asymmetry of funnel plots and Egger's regression test. ${ }^{25}$ We will explore the potential small-study effects in the network using a comparison-adjusted funnel plot. $^{31}$

\section{Methods for direct treatment comparisons}

To supplement NMA, direct estimates will be reported and estimated using pairwise meta-analysis. Pairwise meta-analyses for direct treatment comparisons (at least two RCTs) 
of primary and secondary outcomes will be performed using the random-effects model (Review Manager V.5.3 Nordic Cochrane Centre, The Cochrane Collaboration). We chose to use the random-effects model as it accounts for the differences between populations included in the different trials. ${ }^{25}$ Effect estimates and $95 \%$ CI will be calculated for our outcomes. We will report dichotomous variables as percentages while continuous variables will be reported as mean \pm SD deviation or median (IQR).

\section{Assessment of heterogeneity}

We will qualitatively assess extent of heterogeneity by comparing the study population characteristics, interventions and outcome of included trials within each pairwise comparison. To assess extent of methodological heterogeneity, we will qualitatively compare risk of bias within each pairwise comparison. We will assess the statistical heterogeneity within each pairwise comparison by using the $\mathrm{I}^{2}$ index. ${ }^{37}$ Furthermore, if considerable heterogeneity is found, we will attempt to explain heterogeneity in a subgroup analysis or metaregression, when possible. We will assume a common heterogeneity variance $\left(\tau^{2}\right.$ estimated using restricted maximum likelihood approach, across the different comparisons in the network) ${ }^{38-40}$ as the treatments of interest are of the same nature, in which the inhibition of clotting factors is essential for treatment to be efficacious. ${ }^{41}$

\section{Subgroup analysis and metaregression}

We will prespecify the following study-level effect modifiers as potential sources of heterogeneity and inconsistency: CABG type (on/off pump), concomitant antifibrinolytic therapy and timing of drug initiation. Based on available data, we will perform subgroup analyses or metaregression for primary outcomes.

\section{Sensitivity analysis}

We will also perform sensitivity analyses of the primary outcomes to determine the sensitivity of results to changes in the original assumptions. To deal with missing binary data, we will use the best-worst scenario analysis. We will also conduct a series of sensitivity analyses by including only trials with a low risk of bias in all domains and excluding studies with less than $20 \%$ dropout, $20 \%$ to $50 \%$ dropout and greater than $50 \%$ dropout separately to assess the influence of participant dropout in the network.

\section{DISCUSSION}

The evidence basis around the addition of an antithrombotic agent to aspirin after CABG has led to different and divergent interpretations. Indeed, although several studies have shown benefits in terms of improving shortterm and long-term SVG patency, the overall literature has not yet been able to show a clearly beneficial clinical effect without the cost of an increased risk of bleeding complications with dual antithrombotic therapy. This study aims to qualitatively and quantitatively synthesise the available evidence of comparative efficacy and safety of different antithrombotic agents in patients undergoing CABG. We will also present the relative ranking of each competing treatment for our outcomes of interest. To the best of our knowledge, this will be the first review to simultaneously evaluate the relative effects of multiple antithrombotic agents among CABG patients using an NMA approach.

Our review has several strengths. We will conduct a comprehensive literature search to identify both published and unpublished studies; restriction of studies to RCTs; duplicate assessment of eligibility, risk of bias and data abstraction. Moreover, we will use GRADE to assess the quality of included studies. Some limitations are predictable. First, excluding non-English studies may introduce publication bias. We also expect to encounter a considerable range of publication dates for the studies, and thus, contemporaneous surgical techniques and treatment comparisons may not reflect the current clinical practice. If the number of included studies is small, the ability to explore heterogeneity, conduct metaregression and even perform NMA could be limited. If the degree of clinical heterogeneity is substantial and the distributions of important effect modifiers across treatment comparisons are not balanced, the internal validity may be threatened. Finally, due to the study-level nature of our review, the lack of individual participant data will preclude performing a more robust analysis to explore differences in patient-level covariates.

The results of this NMA will inform clinicians, patients and guideline developers the best available evidence on relative benefits of antithrombotic agents after CABG while considering the side effect profile. We also expect that our findings will significantly advance the knowledge around the relative ranking of antithrombotic agents, will fill the gaps, as well as trigger further research to enhance clinical decision-making and patient-important outcomes.

\section{ETHICS AND DISSEMINATION}

Due to the nature of the study, there are no ethical concerns nor informed consent required. We will disseminate the results of our systematic review and NMA through a peer-reviewed journal.

\section{Author affiliations}

${ }^{1}$ Department of Epidemiology and Biostatistics, Schulich School of Medicine and Dentistry, Western University, London, Ontario, Canada

${ }^{2}$ Department of Anesthesia and Perioperative Medicine, Centre for Medical Evidence, Decision Integrity \& Clinical Impact (MEDICI), Western University, London, Ontario, Canada

${ }^{3}$ Division of Cardiology, London Health Sciences Centre, London, Ontario, Canada ${ }^{4}$ Epidemiology Division, University of Toronto, Dalla Lana School of Public Health, Toronto, Ontario, Canada

${ }^{5}$ Interfaculty Program in Public Health, Western University, London, Ontario, Canada ${ }^{6} \mathrm{Health}$ Quality Ontario, London, Ontario, Canada

${ }^{7}$ Keele Cardiovascular Research Group, Institute for Applied Clinical Science and Centre for Prognosis Research, Institute of Primary Care and Health Sciences, University of Keele, Keele, UK 
Contributors KS and RB conceived and designed the study, drafted and critically reviewed the manuscript and approved the final manuscript as submitted. SL and MAM were involved in the concept and design of the study, critically reviewed and edited manuscript and approved the final manuscript as submitted. JM, AJ-B and TC were involved in the concept and design of the study, critically reviewed and approved the final manuscript as submitted. CK and IFN were involved in the concept and design of the study, provided health-research methodology and statistical insight, critically reviewed and approved the final manuscript as submitted. SL, MAM, TC and RB provided expertise on cardiovascular outcomes, antithrombotics and coronary artery disease. All authors contributed to the development of the selection criteria. All authors read, provided feedback and approved the final manuscript as submitted. RB is the guarantor.

Funding This research received no specific grant from any funding agency in the public, commercial or not-for-profit sectors.

Competing interests None declared.

Patient consent Not required.

Provenance and peer review Not commissioned; externally peer reviewed.

Open Access This is an Open Access article distributed in accordance with the Creative Commons Attribution Non Commercial (CC BY-NC 4.0) license, which permits others to distribute, remix, adapt, build upon this work non-commercially, and license their derivative works on different terms, provided the original work is properly cited and the use is non-commercial. See: http://creativecommons.org/ licenses/by-nc/4.0/

(c) Article author(s) (or their employer(s) unless otherwise stated in the text of the article) 2018. All rights reserved. No commercial use is permitted unless otherwise expressly granted.

\section{REFERENCES}

1. Hillis LD, Smith PK, Anderson JL, et al. 2011 ACCF/AHA Guideline for Coronary Artery Bypass Graft Surgery: a report of the American College of Cardiology Foundation/American Heart Association Task Force on Practice Guidelines. Circulation 20112011;124:e652-735.

2. Kulik A, Ruel $M$, Jneid $H$, et al. Secondary prevention after coronary artery bypass graft surgery: a scientific statement from the American Heart Association. Circulation 2015;131:927-64.

3. Fitzgibbon GM, Kafka HP, Leach AJ, et al. Coronary bypass graft fate and patient outcome: angiographic follow-up of 5,065 grafts related to survival and reoperation in 1,388 patients during 25 years. J Am Coll Cardiol 1996;28:616-26.

4. Halabi AR, Alexander JH, Shaw LK, et al. Relation of early saphenous vein graft failure to outcomes following coronary artery bypass surgery. Am J Cardiol 2005;96:1254-9.

5. Lopes RD, Mehta RH, Hafley GE, et al. Relationship between vein graft failure and subsequent clinical outcomes after coronary artery bypass surgery. Circulation 2012;125:749-56.

6. Cooper GJ, Underwood MJ, Deverall PB. Arterial and venous conduits for coronary artery bypass a current review. Eur $J$ Cardiothorac Surg 1996;10:129-40.

7. Campeau L. Failure of saphenous vein coronary artery bypass grafts and its potential prevention. Curr Opin Cardiol 1987;2:990-5.

8. Goldman S, Zadina K, Moritz T, et al. Long-term patency of saphenous vein and left internal mammary artery grafts after coronary artery bypass surgery: results from a Department of Veterans Affairs Cooperative Study. J Am Coll Cardiol 2004;44:2149-56.

9. de Vries MR, Simons KH, Jukema JW, et al. Vein graft failure: from pathophysiology to clinical outcomes. Nat Rev Cardiol 2016;13:451-70.

10. Chesebro JH, Fuster V, Elveback LR, et al. Effect of dipyridamole and aspirin on late vein-graft patency after coronary bypass operations. N Engl J Med 1984;310:209-14.

11. Lorenz RL, Schacky CV, Weber M, et al. Improved aortocoronary bypass patency by low-dose aspirin (100 mg daily). Effects on platelet aggregation and thromboxane formation. Lancet 1984;1:1261-4.

12. Goldman S, Copeland J, Moritz T, et al. Improvement in early saphenous vein graft patency after coronary artery bypass surgery with antiplatelet therapy: results of a Veterans Administration Cooperative Study. Circulation 1988;77:1324-32.

13. Goldman S, Copeland J, Moritz T, et al. Saphenous vein graft patency 1 year after coronary artery bypass surgery and effects of antiplatelet therapy. Results of a Veterans Administration Cooperative Study. Circulation 1989;80:1190-7.
14. Dacey LJ, Munoz JJ, Johnson ER, et al. Effect of preoperative aspirin use on mortality in coronary artery bypass grafting patients. Ann Thorac Surg 2000;70:1986-90.

15. Mangano DT. Multicenter Study of Perioperative Ischemia Research Group. Aspirin and mortality from coronary bypass surgery. $N$ Engl J Med 2002;347:1309-17.

16. Bybee KA, Powell BD, Valeti U, et al. Preoperative aspirin therapy is associated with improved postoperative outcomes in patients undergoing coronary artery bypass grafting. Circulation 2005;112(9 Suppl):I286-92.

17. Stein PD, Schünemann HJ, Dalen JE, et al. Antithrombotic therapy in patients with saphenous vein and internal mammary artery bypass grafts: the Seventh ACCP conference on antithrombotic and thrombolytic therapy. Chest 2004;126:600S-8.

18. Gao C, Ren C, Li D, et al. Clopidogrel and aspirin versus clopidogrel alone on graft patency after coronary artery bypass grafting. Ann Thorac Surg 2009;88:59-62.

19. Gao G, Zheng Z, Pi Y, et al. Aspirin plus clopidogrel therapy increases early venous graft patency after coronary artery bypass surgery a single-center, randomized, controlled trial. J Am Coll Cardiol 2010;56:1639-43.

20. Kulik A, Le May MR, Voisine P, et al. Aspirin plus clopidogrel versus aspirin alone after coronary artery bypass grafting: the Clopidogrel After Surgery for Coronary Artery Disease (CASCADE) Trial. Circulation 2010;122:2680-7.

21. Sun JC, Teoh KH, Lamy A, et al. Randomized trial of aspirin and clopidogrel versus aspirin alone for the prevention of coronary artery bypass graft occlusion: the Preoperative Aspirin and Postoperative Antiplatelets in Coronary Artery Bypass Grafting study. Am Heart J 2010;160:1178-84.

22. Mannacio VA, Di Tommaso L, Antignan A, et al. Aspirin plus clopidogrel for optimal platelet inhibition following off-pump coronary artery bypass surgery: results from the CRYSSA (prevention of Coronary arteRY bypaSS occlusion After off-pump procedures) randomised study. Heart 2012;98:1710-5.

23. Zimmermann N, Gams E, Hohlfeld T. Aspirin in coronary artery bypass surgery: new aspects of and alternatives for an old antithrombotic agent. Eur J Cardiothorac Surg 2008;34:93-108.

24. Caldwell DM, Ades AE, Higgins JP. Simultaneous comparison of multiple treatments: combining direct and indirect evidence. BMJ 2005;331:897-900.

25. Higgins JPT, Se G. Cochrane handbook for systematic reviews of interventions version 5.1.0 [updated March 2011]: the cochrane collaboration. $2011 \mathrm{http}: / /$ handbook.cochrane.org

26. Hutton B, Salanti G, Caldwell DM, et al. The PRISMA extension statement for reporting of systematic reviews incorporating network meta-analyses of health care interventions: checklist and explanations. Ann Intern Med 2015;162:777-84.

27. Chesebro JH, Knatterud G, Roberts R, et al. Thrombolysis in Myocardial Infarction (TIMI) Trial, Phase I: a comparison between intravenous tissue plasminogen activator and intravenous streptokinase. Clinical findings through hospital discharge. Circulation 1987;76:142-54.

28. Higgins JPT, Sterne JAC, Savović J, et al. A revised tool for assessing risk of bias in randomized trials. In: Chandler J, McKenzie J, Boutron I, Welch V, editors. Cochrane methods. Cochrane Database Syst Rev 2016;10(Suppl 1):29-31.

29. Puhan MA, Schünemann HJ, Murad MH, et al. A GRADE Working Group approach for rating the quality of treatment effect estimates from network meta-analysis. BMJ 2014;349:g5630.

30. Higgins JP, Jackson D, Barrett JK, et al. Consistency and inconsistency in network meta-analysis: concepts and models for multi-arm studies. Res Synth Methods 2012;3:98-110.

31. Chaimani A, Higgins JP, Mavridis D, et al. Graphical tools for network meta-analysis in STATA. PLoS One 2013;8:e76654.

32. Jansen JP, Schmid CH, Salanti G. Directed acyclic graphs can help understand bias in indirect and mixed treatment comparisons. J Clin Epidemiol 2012;65:798-807.

33. Kabali C, Ghazipura M. Transportability in network meta-analysis Epidemiology 2016;27:556-61.

34. StataCorp. Stata statistical software: release 14. College Station, TX: StataCorp LP, 2015.

35. Salanti G, Ades AE, loannidis JP. Graphical methods and numerical summaries for presenting results from multiple-treatment metaanalysis: an overview and tutorial. J Clin Epidemiol 2011;64:163-71.

36. Salanti G. Indirect and mixed-treatment comparison, network, or multiple-treatments meta-analysis: many names, many benefits, many concerns for the next generation evidence synthesis tool. Res Synth Methods 2012;3:80-97.

37. Higgins JP, Thompson SG, Deeks JJ, et al. Measuring inconsistency in meta-analyses. BMJ 2003;327:557-60. 
38. Higgins JP, Whitehead A. Borrowing strength from external trials in a meta-analysis. Stat Med 1996;15:2733-49.

39. Dias S, Welton NJ, Sutton AJ, et al. Evidence synthesis for decision making 4: inconsistency in networks of evidence based on randomized controlled trials. Med Decis Making 2013;33:641-56.
40. Lumley T. Network meta-analysis for indirect treatment comparisons Stat Med 2002;21:2313-24.

41. Mega JL, Simon T. Pharmacology of antithrombotic drugs: an assessment of oral antiplatelet and anticoagulant treatments. Lancet 2015;386:281-91. 